\title{
Modeling the Effect of Inoculum Size on the Thermal Inactivation of Salmonella Typhimurium to Elimination in Ground Chicken Thigh Meat
}

\author{
Thomas P. Oscar* \\ Department of Agriculture, Agricultural Research Service, Residue Chemistry and Predictive Microbiology Research Unit, Center for \\ Food Science and Technology, University of Maryland Eastern Shore, Princess Anne, Maryland, U.S. \\ *Corresponding author: thomas.oscar@ars.usda.gov
}

\begin{abstract}
A study was undertaken to investigate and model the effect of inoculum size on the thermal inactivation of Salmonella to elimination in ground chicken by conduction heating. To develop the model, ground chicken thigh meat portions $\left(0.76 \mathrm{~cm}^{3}\right)$ in microcentrifuge tubes were inoculated with $2.0,3.6$, or $5.2 \log$ of a single strain of Salmonella Typhimurium followed by cooking for 0 to $10 \mathrm{~min}$ at 52 to $100^{\circ} \mathrm{C}$ in a heating block. To validate the model, the ground chicken portions were inoculated with 2.8 or $4.4 \log$ of $S$. Typhimurium followed by cooking for 0 to $9 \mathrm{~min}$ at 55 to $97^{\circ} \mathrm{C}$. An automated, whole sample enrichment, miniature most probable number (MPN) method with a lower limit of detection of one Salmonella cell per portion was used for enumeration. The MPN data were used to develop $(n=851)$ and validate $(n=256)$ a multiple layer feedforward neural network model with two hidden layers of two nodes each. Model performance was evaluated using the acceptable prediction zone (APZ) method. The proportion of residuals in an APZ (pAPZ) from - $1 \log$ (fail-safe) to $0.5 \log$ (fail-dangerous) was 0.945 (804/851) for dependent data and $0.945(242 / 256)$ for independent data for interpolation. A pAPZ $\geq 0.7$ indicated that model predictions had acceptable bias and accuracy. Thus, the model was successfully validated. The time for elimination of Salmonella at $58^{\circ} \mathrm{C}$ was 5.6, 7.1, and $8.7 \mathrm{~min}$ for inoculum sizes of 2.0, 3.6 and 5.2 log per portion, respectively. This relationship was observed for all cooking temperatures and among all inoculum sizes investigated indicating that inoculum size was an important independent variable to include in the model.
\end{abstract}

Keywords: ground chicken, Salmonella Typhimurium, thermal inactivation, neural network, modeling, inoculum size, validation

Cite This Article: Thomas P. Oscar, "Modeling the Effect of Inoculum Size on the Thermal Inactivation of Salmonella Typhimurium to Elimination in Ground Chicken Thigh Meat." American Journal of Food Science and Technology, vol. 5, no. 4 (2017): 135-142. doi: 10.12691/ajfst-5-4-3.

\section{Introduction}

The thermal inactivation of Salmonella in chicken has been extensively investigated, modeled, and reviewed $[1,2,3]$. There are multiple variables in addition to time and temperature that affect the kinetics of Salmonella death during cooking of chicken. For example, composition of chicken meat and meat products [4,5], strain of Salmonella [6,7], and size and shape of chicken meat and meat products [7]. One variable that has not received much attention but that could be important is inoculum size. This variable has not been investigated and modeled because it is generally assumed that the kinetics of Salmonella death during the cooking of chicken are independent of the initial number present. However, that is not what was found in the present study.

Several researchers $[8,9,10]$ that have investigated and modeled the thermal inactivation of Salmonella in chicken have used a similar approach. In these studies, a single and high initial number (e.g. $7 \mathrm{log} / \mathrm{g}$ ) of a cocktail of
Salmonella serotypes was inoculated into chicken meat. The inoculated samples were then placed in a plastic bag, heat sealed, and flattened to a thin layer $(1-3 \mathrm{~mm})$. The samples were then cooked in a preheated water bath and cooled rapidly after cooking by immersion in an ice bath. Survivors were then enumerated by spread plating onto a nonselective or selective agar media. Most often, the death kinetics were log-linear, which allowed determination of Dvalues (time for a one $\log$ reduction) and then $z$-values (change in temperature for a one log reduction of the D-value). The D- and z-values were then used in a lethality model to predict the thermal inactivation of Salmonella in chicken under dynamic conditions of heating and cooling. However, this approach does not simulate how chicken is actually cooked and cooled by consumers.

Consequently, a new approach for investigating and modeling the thermal inactivation of Salmonella in chicken meat was developed that more closely simulates how chicken is cooked and cooled by consumers [11]. This new approach involves cooking small cylindrical portions $\left(0.76 \mathrm{~cm}^{3}\right)$ of ground chicken meat in microcentrifuge 
tubes by conduction in a heating block followed by cooling at and to room temperature. After cooling, survivors are enumerated by an automated, whole sample enrichment, miniature most probable number (WSE-mMPN) method. This enumeration method has a lower limit of detection of one Salmonella cell per chicken portion, which allows modeling pathogen death to elimination without extrapolating beyond the data. In the present study, this new approach was used to investigate and model the effect of inoculum size on the thermal inactivation of Salmonella to elimination in ground chicken during cooking.

\section{Materials and Methods}

\subsection{Data Acquisition}

Data for development and validation of the model were acquired in a series of cooking trials using a protocol developed and described in a previous study [11]. In brief, cylindrical portions $\left(0.76 \mathrm{~cm}^{3}\right)$ of ground chicken thigh meat with native microflora in polypropylene microcentrifuge tubes $(1.5 \mathrm{ml})$ were inoculated $(2.0,3.6$, or $5.2 \log )$ with stationary phase cells of a single strain of Salmonella Typhimurium, which was isolated from a chicken breast. The chicken portions were cooked by conduction in a heating block. After cooling at and to room temperature for $30 \mathrm{~min}$, the number of Salmonella remaining in the chicken portions was determined by an automated (serial dilution and transfer steps were performed by a robotic pipettor), whole sample enrichment (WSE), miniature (m) most probable number (MPN) method (3 replicate by 8 dilution) in a $2 \mathrm{ml}, 96$ deep well format.

The WSE-mMPN method has a lower limit of detection of one Salmonella cell per chicken portion and involved incubation $\left(24 \mathrm{~h}\right.$ at $\left.40^{\circ} \mathrm{C}\right)$ in buffered peptone water (to allow injured cells to recover and be quantified) followed by incubation $\left(48 \mathrm{~h}\right.$ at $42^{\circ} \mathrm{C}$ ) in Rappaport Vassiliadis R10 broth (RVB) to detect and enumerate Salmonella survivors. After $48 \mathrm{~h}$ of incubation in RVB, wells that contained Salmonella were white, whereas wells that did not contain Salmonella were blue. This was confirmed by an AOAC-approved lateral flow assay for Salmonella. The number of Salmonella was calculated by the method of Thomas [12]. The temperature of a non-inoculated chicken portion was recorded during cooking trials using a digital thermometer. The initial temperature of the chicken portions was $24.2 \pm 1.9^{\circ} \mathrm{C}$ (mean $\pm \mathrm{SD}, n=140$ trials).

\subsection{Experimental Designs}

Two experiments were conducted. Both used full factorial designs. The first experiment involved three inoculum sizes $(2.0,3.6,5.2 \mathrm{log} /$ portion), eight sampling times $(0,1,2,3,4,6,8,10 \mathrm{~min})$, nine cooking temperatures $\left(52,58,64,70,76,82,88,94,100^{\circ} \mathrm{C}\right)$ and four replicate trials (trial $=$ one combination of the independent variables: inoculum size, cooking temperature, and sampling time) for a total of 108 cooking trials. The data (dependent) from this experiment were used to develop the model.

The second experiment was conducted at intermediate levels of the independent variables used to develop the model and involved two inoculum sizes (2.8, $4.4 \mathrm{log} /$ portion), eight sampling times $(0,0.5,1.5,2.5,3.5,5,7,9 \mathrm{~min})$, eight cooking temperatures $(55,61,67,73,79,85,91$, $97^{\circ} \mathrm{C}$ ), and two replicates for a total of 32 cooking trials. The data (independent) from this experiment were used to evaluate the model for its ability to interpolate.

The data in the second experiment were collected using the same methods as those used to collect the data in the first experiment. This was done so that comparisons of these data to model predictions would not be confounded. In addition, they were collected in such a manner that they provided complete and uniform coverage of model predictions so that a complete and unbiased evaluation of model performance could be obtained.

\subsection{Modeling}

The model consisted of two sub-models. The first sub-model predicted the thermal inactivation of $S$. Typhimurium in ground chicken as function of cooking temperature, inoculum size, and cooking time. To develop this model, a dataset $(n=1,107)$ with the dependent $(n=851)$ and the independent data $(n=256)$ was created in an Excel 2013 spreadsheet (MicroSoft Corp., Redmond, WA). It had five columns: 1) tag; 2) cooking temperature (independent numerical variable); 3) inoculum size (independent numerical variable); 4) cooking time (independent numerical variable); and 5) log number per portion (dependent numerical variable). The tag variable identified the dependent data for model development and the independent data for interpolation.

A spreadsheet add-in program NeuralTools (version 6.3.1, Palisade Corp., Ithaca, NY) was used to develop the model. However, before developing the model, the dependent variable was transformed. Specifically, when the log number per portion was greater than zero, it was normalized by dividing it by the inoculum size with the restriction that the maximum transformed value was one. Samples that tested negative for Salmonella were assigned a value of -1 log per portion for modeling purposes.

A multiple layer feedforward neural network with two hidden layers of two nodes each was then developed as previously described [11]. This model used the hyperbolic tangent function in the hidden layers and the identity function in the output layer. After the neural network was developed, the predict function of NeuralTools was used to construct the sub-model. A formula was included in the sub-model that untransformed the dependent variable so that the sub-model predicted the log number per portion versus time.

The second sub-model was developed in a similar manner. This sub-model predicted the temperature profile at the center of the chicken portion during cooking. As before, a dataset $(n=1,121)$ with the dependent data ( $n=$ $865)$ and the independent data $(n=256)$ was created in an Excel 2013 spreadsheet. However, this dataset only had four columns: 1) tag; 2) cooking temperature (independent numerical variable); 3) cooking time (independent numerical variable); and 4) meat temperature in ${ }^{\circ} \mathrm{C}$ (dependent numerical variable). Again, the tag variable identified the dependent and independent data and NeuralTools was used to develop the sub-model, which was a multiple layer feedforward neural network with a single hidden layer of two nodes. 
For proprietary reasons, NeuralTools does not provide weights and bias values for the neural networks it produces and the current model was too complex to easily develop a stand-alone version of the model like we have done in previous studies $[11,13]$. Thus, users of the current model will need have access to NeuralTools to run it. Nonetheless, once published, the model and the data used to develop and evaluate it will be made available at no cost on our website at www.ars.usda.gov/nea/errc/PoultryFARM.

\subsection{Model Performance}

Performance of the sub-model for thermal inactivation was evaluated using the acceptable prediction zone (APZ) method [14]. In brief, a prediction was considered acceptable when the residual (observed - predicted) was in an APZ from -1 log (fail-safe) to $0.5 \log$ (fail-dangerous). Model predictions for a set of data (whole dataset or data for an individual thermal inactivation curve) were considered to have acceptable bias and accuracy when the proportion of residuals in the APZ (pAPZ) was $\geq 0.7$. The model was considered validated when the independent data for interpolation met the test data criteria (i.e. data were not used in model development, they were collected with the same methods, and they provided uniform and complete coverage of model predictions) of the APZ method, which they did, and the pAPZ for the dependent data and the
pAPZ for the independent data for interpolation were acceptable.

\subsection{Data Analysis}

The validated model was used to determine the predicted time for elimination of Salmonella from the chicken portions. To accomplish this, the cooking temperature and inoculum size of interest were entered into the model and then the time was adjusted until the predicted log number of Salmonella changed from 0.00 to -0.01 . The non-linear portion of the thermal inactivation curve was included in the determination of this kinetic parameter.

\section{Results}

\subsection{Model Predictions}

The model developed is shown in Figure 1. The user enters the cooking temperature in cell E2 and the inoculum size in cell E3 and then the model predicts the thermal inactivation curve, the meat temperature profile, and the cell death profile. The cooking temperature should be a value between 52 and $100^{\circ} \mathrm{C}$, whereas the inoculum size should be a value between 2.0 and $5.2 \log$ per portion. Entering values outside these ranges, which were used to develop and validate the model, is not recommended because it may result in predictions that are not reliable.

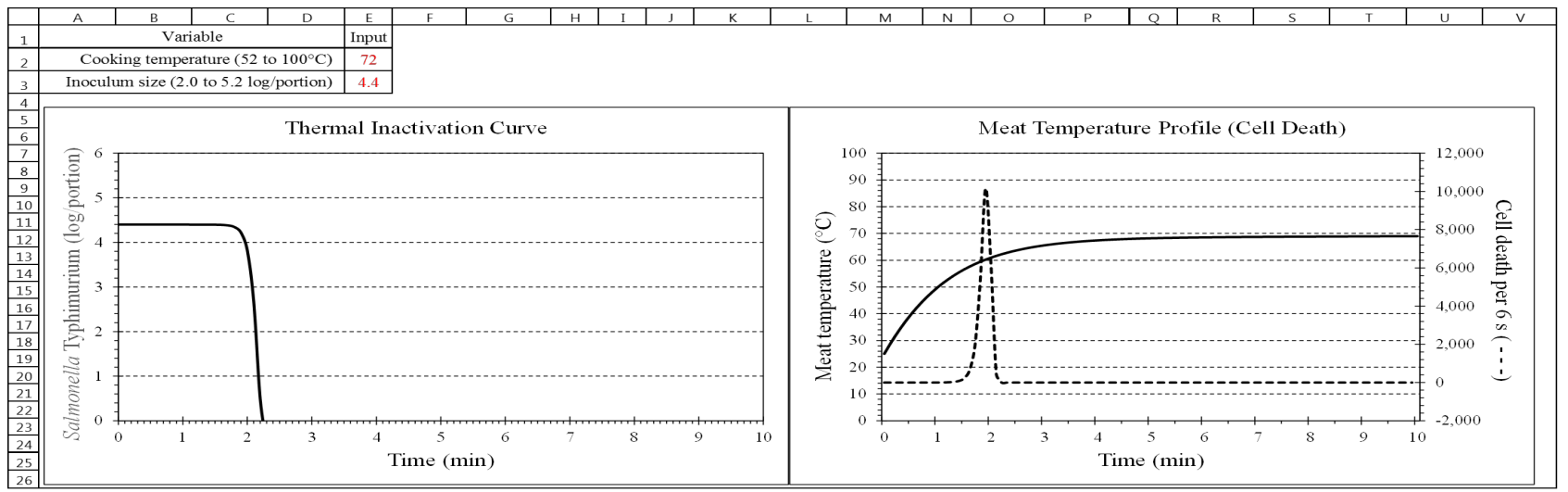

Figure 1. Neural network model that predicts the thermal inactivation, meat temperature profile, and cell death profile of Salmonella Typhimurium in ground chicken thigh meat portions $\left(0.76 \mathrm{~cm}^{3}\right)$ as a function of cooking temperature and inoculum size. Results are for a cooking temperature of $72^{\circ} \mathrm{C}$ and an inoculum size of $4.4 \log$ /portion

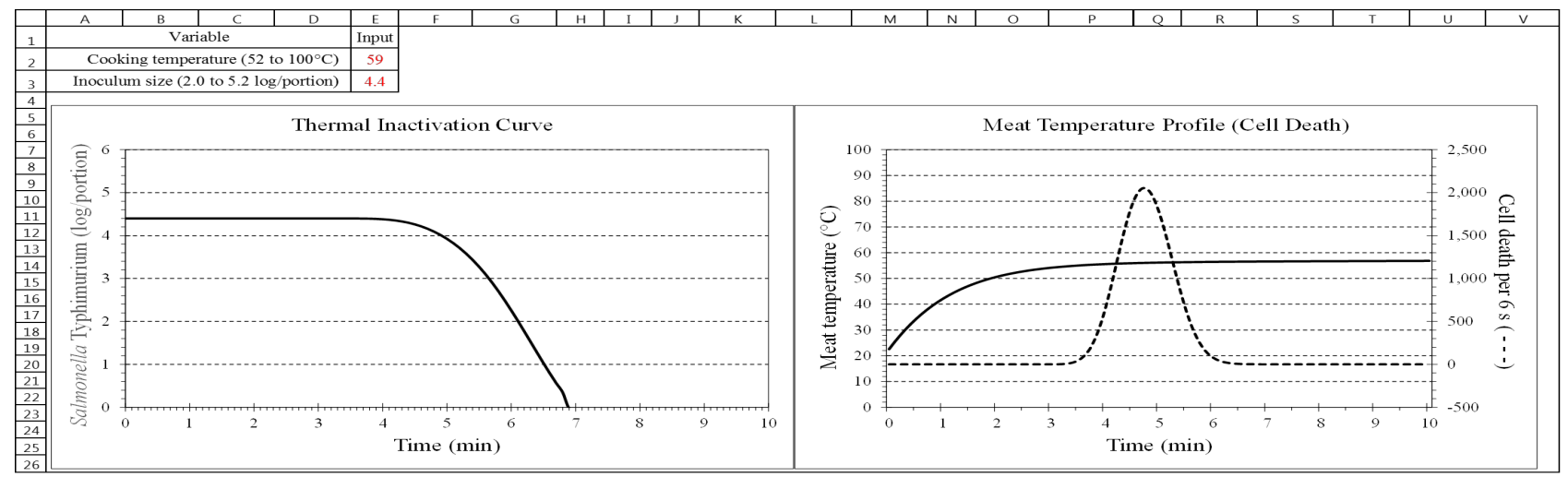

Figure 2. Neural network model that predicts the thermal inactivation, meat temperature profile, and cell death profile of Salmonella Typhimurium in ground chicken thigh meat portions $\left(0.76 \mathrm{~cm}^{3}\right)$ as a function of cooking temperature and inoculum size. Results are for a cooking temperature of $59^{\circ} \mathrm{C}$ and an inoculum size of $4.4 \mathrm{log} /$ portion 
The results in Figure 1 are for a cooking temperature of $72^{\circ} \mathrm{C}$ and an inoculum size of $4.4 \mathrm{log}$ per portion. The temperature of the center of the chicken portion, which was $5 \mathrm{~mm}$ from the heat source, increased during cooking in a non-linear manner until it plateaued around five minutes of cooking. There was no initial change in the number of Salmonella (lag phase) until the chicken warmed to temperatures that caused inactivation. Once inactivation started at around $1.5 \mathrm{~min}$ it proceeded quickly with total elimination of Salmonella by $2.3 \mathrm{~min}$ of cooking.

The model predictions in Figure 2 are for a cooking temperature of $59^{\circ} \mathrm{C}$ and an inoculum size of $4.4 \mathrm{log}$ per portion. At this lower cooking temperature the inactivation of Salmonella started around $3.6 \mathrm{~min}$ and took longer to complete around $6.9 \mathrm{~min}$.

\subsection{Model Performance}

The first step in evaluating the data acquired for model development and validation involved graphing the mean observed values and the predicted values as a function of the independent variables (i.e. temperature, time, and inoculum size). A representative sample of the 17 graphs prepared are shown in Figure 3. A few general observations can be made. First, the meat temperature profile during cooking followed a similar pattern across all
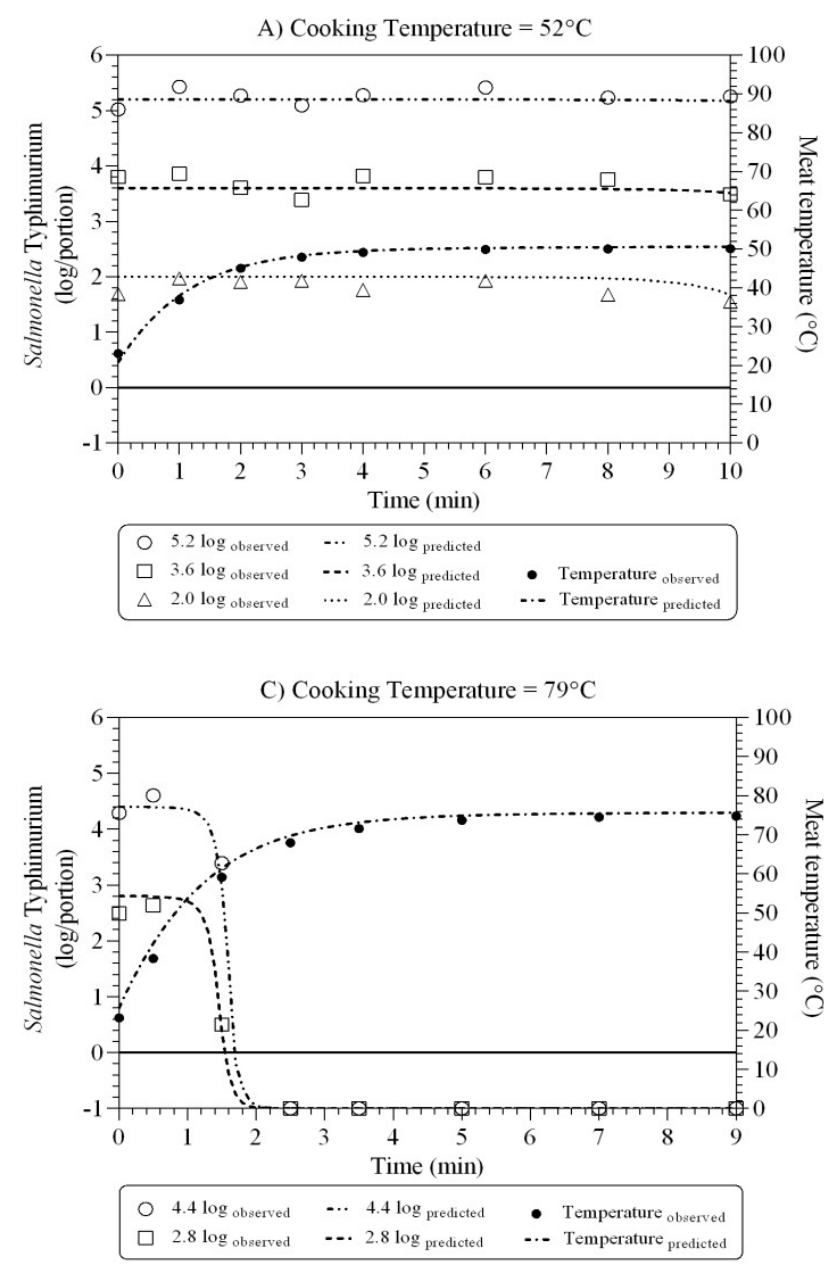

cooking temperatures investigated. More specifically, the temperature at the center of the chicken portion increased in a non-linear manner over time until it plateaued at about 5 min of cooking. Second, the final temperature of the chicken portion at 10 min of cooking was always slightly below the cooking temperature (Table 1). Third, at all cooking temperatures there was an initial phase where the number of Salmonella did not change (lag phase), which lasted until the chicken portion warmed to temperatures that were high enough to kill the pathogen. Once lag phase ended, death was rapid; especially at higher cooking temperatures.

The second step of evaluating the data acquired for model development and validation was to compare the observed and predicted values (log number per portion) using the acceptable prediction zone (APZ) method. A representative sample of the 17 residual plots prepared are shown in Figure 4. The proportion of residuals in the acceptable prediction zone (pAPZ) was calculated for each thermal inactivation curve and found to range from 0.75 to 1 (Table 1$)$. Overall, the pAPZ was $0.945(804 / 851)$ for dependent data and 0.945 (242/256) for independent data for interpolation. A pAPZ of 0.7 or higher indicated that the model provided predictions with acceptable bias and accuracy. Thus, the model was successfully validated because it provided acceptable predictions for both the dependent data and the independent data for interpolation.
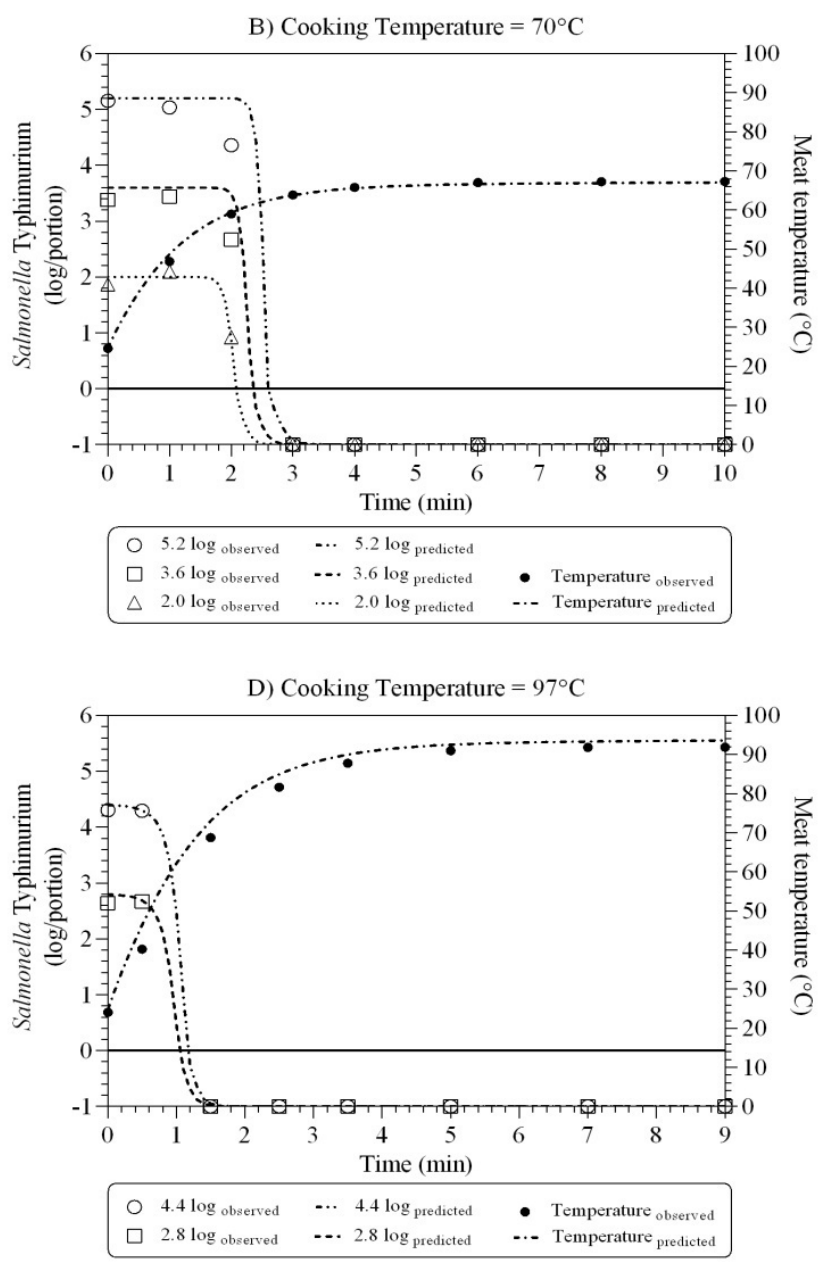

Figure 3. Thermal inactivation curves for Salmonella Typhimurium in ground chicken thigh meat portions $\left(0.76 \mathrm{~cm}^{3}\right)$ and meat temperature profiles during cooking at A) $52^{\circ} \mathrm{C}$ (dependent data); B) $70^{\circ} \mathrm{C}$ (dependent data); C) $79^{\circ} \mathrm{C}$ (independent data for interpolation); and D) $97^{\circ} \mathrm{C}$ (independent data for interpolation) and as a function of inoculum size. Symbols are mean observed values and lines are predicted values 
Table 1. Effect of cooking temperature and inoculum size on predicted final meat temperature, predicted time for elimination, and model performance for thermal inactivation of Salmonella Typhimurium in ground chicken thigh meat for dependent and independent data

\begin{tabular}{|c|c|c|c|c|c|c|c|}
\hline \multirow{2}{*}{ Type of data } & \multirow{2}{*}{$\begin{array}{l}\text { Cooking temperature } \\
\left({ }^{\circ} \mathrm{C}\right)\end{array}$} & \multirow{2}{*}{$\begin{array}{c}\text { Inoculum size } \\
\text { (log/portion) }\end{array}$} & \multirow{2}{*}{$\begin{array}{l}\text { Predicted final meat } \\
\text { temperature }\left({ }^{\circ} \mathrm{C}\right)\end{array}$} & \multirow{2}{*}{$\begin{array}{l}\text { Predicted time for } \\
\text { elimination (min) }\end{array}$} & \multicolumn{3}{|c|}{ Model performance } \\
\hline & & & & & In & Total & $\mathrm{pAPZ}^{\mathrm{a}}$ \\
\hline \multirow[t]{27}{*}{ Dependent } & 52 & 5.2 & 50.6 & $>10$ & 29 & 32 & 0.906 \\
\hline & & 3.6 & & $>10$ & 32 & 32 & 1.000 \\
\hline & & 2.0 & & $>10$ & 32 & 32 & 1.000 \\
\hline & 58 & 5.2 & 55.9 & 8.7 & 24 & 30 & 0.800 \\
\hline & & 3.6 & & 7.1 & 29 & 32 & 0.906 \\
\hline & & 2.0 & & 5.6 & 29 & 32 & 0.906 \\
\hline & 64 & 5.2 & 61.4 & 3.9 & 26 & 31 & 0.839 \\
\hline & & 3.6 & & 3.4 & 29 & 32 & 0.906 \\
\hline & & 2.0 & & 3.0 & 28 & 32 & 0.875 \\
\hline & 70 & 5.2 & 67.1 & 2.6 & 30 & 31 & 0.968 \\
\hline & & 3.6 & & 2.4 & 30 & 32 & 0.938 \\
\hline & & 2.0 & & 2.1 & 31 & 32 & 0.969 \\
\hline & 76 & 5.2 & 72.8 & 2.0 & 29 & 31 & 0.935 \\
\hline & & 3.6 & & 1.8 & 30 & 31 & 0.968 \\
\hline & & 2.0 & & 1.6 & 32 & 32 & 1.000 \\
\hline & 82 & 5.2 & 78.7 & 1.7 & 30 & 31 & 0.968 \\
\hline & & 3.6 & & 1.5 & 32 & 32 & 1.000 \\
\hline & & 2.0 & & 1.4 & 31 & 31 & 1.000 \\
\hline & 88 & 5.2 & 84.6 & 1.5 & 31 & 32 & 0.969 \\
\hline & & 3.6 & & 1.3 & 29 & 32 & 0.906 \\
\hline & & 2.0 & & 1.2 & 31 & 31 & 1.000 \\
\hline & 94 & 5.2 & 90.6 & 1.3 & 31 & 31 & 1.000 \\
\hline & & 3.6 & & 1.2 & 29 & 30 & 0.967 \\
\hline & & 2.0 & & 1.1 & 31 & 32 & 0.969 \\
\hline & 100 & 5.2 & 96.7 & 1.2 & 29 & 31 & 0.935 \\
\hline & & 3.6 & & 1.1 & 29 & 32 & 0.906 \\
\hline & & 2.0 & & 0.9 & 31 & 32 & 0.969 \\
\hline \multirow[t]{16}{*}{ Independent } & 55 & 4.4 & 53.3 & $>10$ & 16 & 16 & 1.000 \\
\hline & & 2.8 & & 9.5 & 14 & 16 & 0.875 \\
\hline & 61 & 4.4 & 58.6 & 5.0 & 14 & 16 & 0.875 \\
\hline & & 2.8 & & 4.1 & 16 & 16 & 1.000 \\
\hline & 67 & 4.4 & 64.2 & 3.0 & 12 & 16 & 0.750 \\
\hline & & 2.8 & & 2.7 & 15 & 16 & 0.938 \\
\hline & 73 & 4.4 & 69.8 & 2.2 & 16 & 16 & 1.000 \\
\hline & & 2.8 & & 1.9 & 16 & 16 & 1.000 \\
\hline & 79 & 4.4 & 75.7 & 1.7 & 15 & 16 & 0.938 \\
\hline & & 2.8 & & 1.6 & 14 & 16 & 0.875 \\
\hline & 85 & 4.4 & 81.6 & 1.5 & 14 & 16 & 0.875 \\
\hline & & 2.8 & & 1.4 & 16 & 16 & 1.000 \\
\hline & 91 & 4.4 & 87.6 & 1.3 & 16 & 16 & 1.000 \\
\hline & & 2.8 & & 1.2 & 16 & 16 & 1.000 \\
\hline & 97 & 4.4 & 93.6 & 1.2 & 16 & 16 & 1.000 \\
\hline & & 2.8 & & 1.1 & 16 & 16 & 1.000 \\
\hline
\end{tabular}

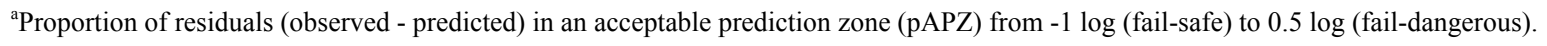



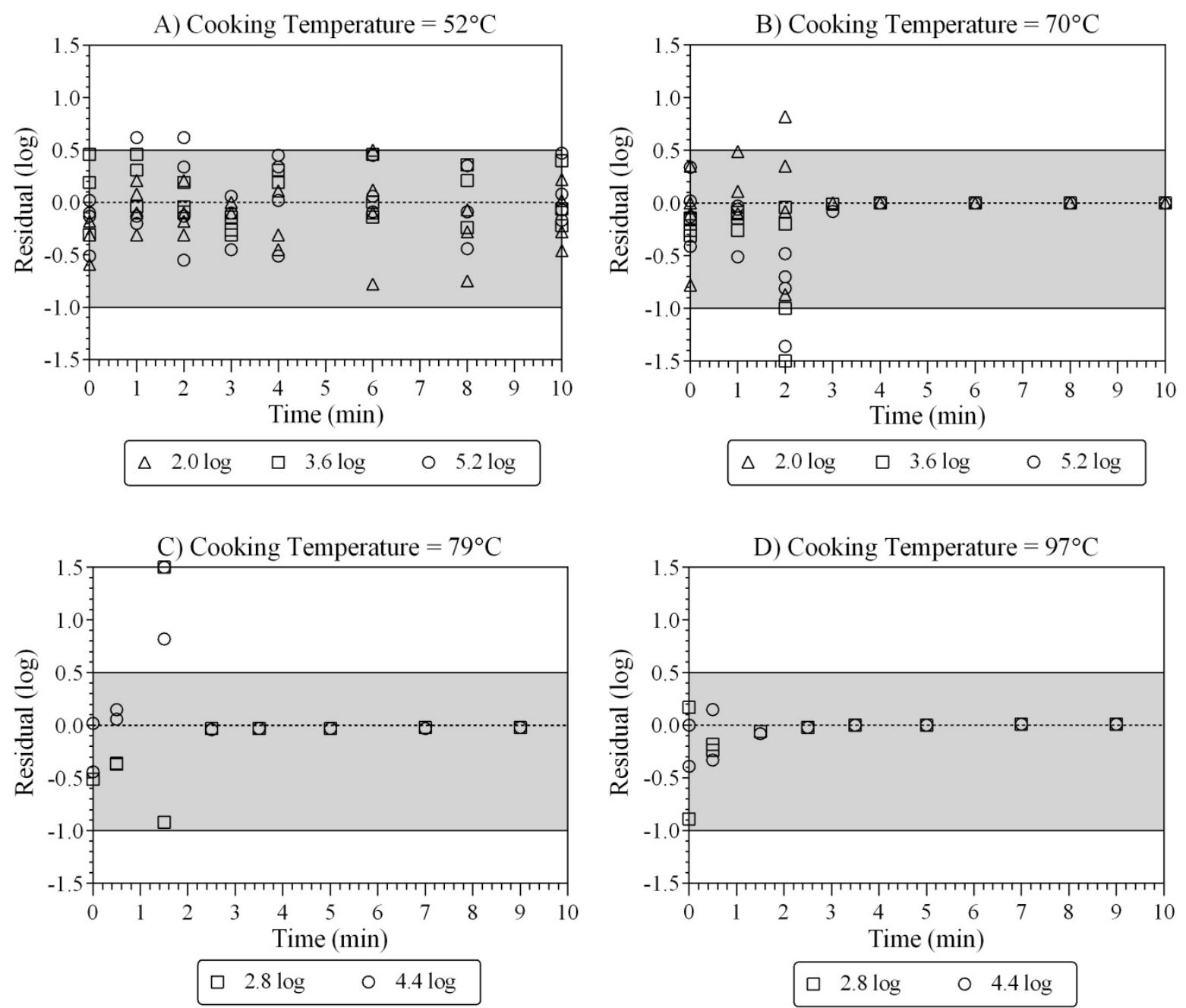

Figure 4. Performance of the model for thermal inactivation of Salmonella Typhimurium in ground chicken thigh meat portions $\left(0.76 \mathrm{~cm}^{3}\right)$ at cooking temperatures of A) $52^{\circ} \mathrm{C}$ (dependent data); B) $70^{\circ} \mathrm{C}$ (dependent data); C) $79^{\circ} \mathrm{C}$ (independent data for interpolation); and D) $97^{\circ} \mathrm{C}$ (independent data for interpolation) and as a function of inoculum size. The acceptable prediction zone (grey box) was from -1 $\log$ (fail-safe) to 0.5 log (fail-dangerous). Residuals $>1.5 \log$ were graphed as $1.5 \log$ for clarity of presentation

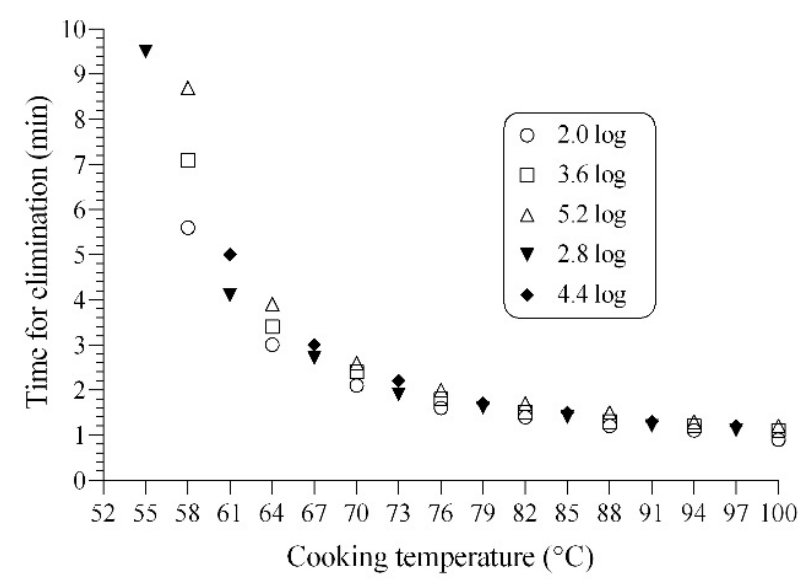

Figure 5. Predicted time for elimination of Salmonella Typhimurium in ground chicken thigh meat portions $\left(0.76 \mathrm{~cm}^{3}\right)$ as a function of cooking temperature and inoculum size

The third step for evaluating the data used in model development and validation involved determining the predicted time for elimination of Salmonella from the chicken portions during cooking. These values (Table 1) were found to decrease in a non-linear manner as the cooking temperature increased (Figure 5). In addition, regardless of cooking temperature, the time for elimination increased as the inoculum size increased. For example, at $58^{\circ} \mathrm{C}$, the time for elimination was 5.6, 7.1, and $8.7 \mathrm{~min}$ for inoculum sizes of 2.0, 3.6, and $5.2 \mathrm{log}$ per portion, respectively. These results indicated that inoculum size was an important independent variable to include in the model.

\section{Discussion}

Bellara et al. [15] investigated the thermal inactivation of Salmonella Typhimurium in agar cylinders heated at $70^{\circ} \mathrm{C}$ in a water bath. To enumerate survivors they sliced open the agar cylinder, incubated the slices, and then quantified the radius of Salmonella growth using image analysis. They found that the radius of growth decreased as a function of time of heating until all of the Salmonella were eliminated. Similar to the current study, the temperature profile at the center of the agar cylinder during heating increased in a non-linear manner and then plateaued. Likewise, a lag period was observed in the thermal inactivation curve until the agar cylinder warmed to temperatures that could kill Salmonella. Once this 
critical point was reached, Salmonella death occurred rapidly just like in the present study. Thus, the general observations concerning the thermal inactivation of Salmonella Typhimurium in cylinders of ground chicken thigh meat in this study were similar to those reported by Bellara et al. [15] for thermal inactivation of Salmonella Typhimurium in agar cylinders.

Mackey et al. [16] used the agar cylinder method to investigate the effect of inoculum size on the thermal inactivation of Salmonella Typhimurium. Similar to the current study, the time for elimination of Salmonella in agar cylinders heated at $70^{\circ} \mathrm{C}$ in a water bath increased as inoculum size increased. More specifically, the time for elimination increased from 30.5 to $33.0 \mathrm{~min}$ as inoculum size increased from 3 to $7 \mathrm{log} / \mathrm{ml}$. In comparison, in the present study, the time for elimination of Salmonella in cylinders of ground chicken thigh meat heated at $70^{\circ} \mathrm{C}$ increased from 2.1 to $2.6 \mathrm{~min}$ as inoculum size increased from 2 to $5.6 \mathrm{log} /$ portion. This finding makes sense as it should take longer for more cells to die under the same conditions of heating. In other words, it should take longer for 1,000 cells to die than 100 cells.

A challenge to developing and validating a model for thermal inactivation of Salmonella to elimination in ground chicken thigh meat with native microflora as a function of inoculum size and a broad range of cooking temperatures and times is that it requires a large amount of enumeration data. Obtaining this type of data in food with native microflora is very time consuming and labor intensive. However, by using an automated, WSE-mMPN method that determines presence/absence of Salmonella as well as number in chicken with native microflora, it was possible to acquire the large amount of enumeration data $(n=1,107)$ needed to develop and validate the model. In fact, all of the data acquired in the present study were collected by a single undergraduate student working parttime for a period of about nine months.

The novel aspects of the current approach to investigating and modeling thermal inactivation of Salmonella in chicken with native microflora were discussed in a previous study [11]. In brief, the novel aspects were: 1) use of WSE-mMPN to determine presence, absence, and number of Salmonella survivors; 2) modeling the data using a neural network approach that simplified data modeling to a single step saving time and money; 3) validation of the model against independent data for interpolation; 4) acquiring and modeling data under dynamic conditions of heating; and 5) developing a model that predicts both thermal inactivation and the meat temperature profile and now the cell death profile.

The limitations of the modeling approach that were previously discussed [11] were: 1) using a single serotype of Salmonella; 2) using one inoculum size, which was addressed in the present study; 3) using a single initial temperature (room temperature); 4) using one distance (5 $\mathrm{mm}$ ) from the heat source; 5) using a maximum cooking temperature of $100^{\circ} \mathrm{C}$, which is below temperatures actually used to pan fry ground chicken; and 6) using a single formulation of chicken meat (thigh meat).

The previous paper [11] also discussed in some detail the difference in the current data collection and modeling approach from that of most other studies in this field. This included: 1) using WSE-mMPN instead of viable counts to enumerate survivors; 2) inoculation of samples with a single strain instead of a cocktail of strains; 3) acquiring data under dynamic temperature conditions instead of constant temperature conditions; 4) having come-up time versus no come-up time; 5) cooling cooked samples at room temperature versus in ice; 5) modeling the data using a neural network versus a multiple step regression approach; and 6) validation of the model against independent data versus no validation.

\section{Conclusion}

The general conclusion being that the current approach simulates better how chicken is cooked and cooled in the real world and thus, results in models that provide more realistic and reliable predictions. However, several important data gaps (see aforementioned limitations) exist in the current models that need to be addressed with further research in order to provide the chicken industry with models that provide even better predictions of the thermal inactivation of Salmonella in chicken with native microflora. The major advance accomplished in the present study was adding inoculum size as an independent variable in the model.

\section{Acknowledgements}

The author appreciates the outstanding technical assistance of Ahmed Abdelrahim (University of Maryland Eastern Shore) without whom this study would not have been successful. Mention of trade names or commercial products is solely for providing specific information and does not imply recommendation or endorsement by the U.S. Department of Agriculture, which is an equal opportunity provider and employer.

\section{References}

[1] Juneja, V.K., "Extrinsic and intrinsic factors that influence microbial heat resistance," Bull. Int. Dairy Fed, 392, 16-30. 2004.

[2] Doyle, M.E., Mazzotta, A.S., "Review of studies on the thermal resistance of Salmonellae," J. Food Prot, 63, 779-795. 2000.

[3] Jarvis, N.A., O'Bryan, C.A., Dawoud, T.M., Park, S.H., Kwon, Y.M., Crandall, P.G., Ricke, S.C., "An overview of Salmonella thermal destruction during food processing and preparation," Food Control, 68, 280-290. 2016.

[4] Juneja, V.K., Eblen, B.S., Marks, H.M., "Modeling non-linear survival curves to calculate thermal inactivation of Salmonella in poultry of different fat levels," Int. J. Food Microbiol, 70, 37-51. 2001.

[5] Murphy, R.Y., Duncan, L.K., Johnson, E.R., Davis, M.D., Smith, J.N., "Thermal inactivation D- and Z-values of Salmonella serotypes and Listeria innocua in chicken patties, chicken tenders, franks, beef patties, and blended beef and turkey patties," J. Food Prot, 65(1), 53-60. 2002.

[6] Juneja, V.K., Eblen, B.S., Ransom, G.M., "Thermal inactivation of Salmonella spp. in chicken broth, beef, pork, turkey, and chicken: determination of D- and z-values," J. Food Sci., 66(1), 146-152. 2001.

[7] Murphy, R.Y., Marks, B.P., Johnson, E.R., Johnson, M.G., "Inactivation of Salmonella and Listeria in ground chicken breast meat during thermal processing," J. Food Prot, 62, 980-985. 1999. 
[8] Osaili, T.M., Al-Nabulsi, A.A., Shaker, R.R., Olaimat, A.N., Jaradat, Z.W., Holley, R.A., "Thermal inactivation of Salmonella Typhimurium in chicken shawirma (gyro)," Int. J. Food Microbiol., 166(1), 15-20. 2013.

[9] Murphy, R.Y., Osaili, T., Duncan, L.K., Marcy, J.A., "Effect of sodium lactate on thermal inactivation of Listeria monocytogenes and Salmonella in ground chicken thigh and leg meat," J. Food Prot, 67, 1403-1407. 2004.

[10] Juneja, V.K., Gonzales-Barron, U., Butler, F., Yadav, A.S., Friedman, M., "Predictive thermal inactivation model for the combined effect of temperature, cinnamaldehyde and carvacrol on starvation-stressed multiple Salmonella serotypes in ground chicken," Int. J. Food Microbiol., 165(2), 184-199. 2013.

[11] Oscar, T.P., "Neural network model for thermal inactivation of Salmonella Typhimurium to elimination in ground chicken: acquisition of data by whole sample enrichment, miniature mostprobable-number method," J. Food Prot., 80(1), 104-112. 2017.

[12] Thomas, H.A., "Bacterial densities from fermentation tube tests," J. Am. Water Works Assoc., 34, 572-576. 1942.
[13] Oscar, T.P., "Neural network models for growth of Salmonella serotypes in ground chicken subjected to temperature abuse during cold storage for application in HACCP and risk assessment," Int. J. Food Sci. Technol., 52, 214-221. 2017.

[14] Oscar, T.P., "Development and validation of primary, secondary and tertiary models for predicting growth of Salmonella Typhimurium on sterile chicken," J. Food Prot., 68, 2606-2613. 2005.

[15] Bellara, S.R., Fryer, P.J., McFarlane, C.M., Thomas, C.R., Hocking, P.M., Mackey, B.M., "Visualization and modelling of the thermal inactivation of bacteria in a model food," Appl. Environ. Microbiol., 65(7), 3095-3099. 1999.

[16] Mackey, B.M., Kelly, A.F., Colvin, J.A., Robbins, P.T., Fryer, P.J., "Predicting the thermal inactivation of bacteria in a solid matrix: simulation studies on the relative effects of microbial thermal resistance parameters and process conditions," Int. J. Food Microbiol., 107(3), 295-303. 2006 